\title{
La crise sociale aux Antilles françaises
}

Retour de la question sociale et reflux du politique

\section{Justin Daniel}

\section{OpenEdition}

1 Journals

Édition électronique

URL : http://journals.openedition.org/echogeo/11117

DOI : 10.4000/echogeo.11117

ISSN : 1963-1197

\section{Éditeur}

Pôle de recherche pour l'organisation et la diffusion de l'information géographique (CNRS UMR 8586)

Référence électronique

Justin Daniel, «La crise sociale aux Antilles françaises », EchoGéo [En ligne], Sur le Vif, mis en ligne le 30 mars 2009, consulté le 01 mai 2019. URL : http://journals.openedition.org/echogeo/11117 ; DOI : 10.4000/echogeo.11117

Ce document a été généré automatiquement le 1 mai 2019.

\section{(c) (i) (3)}

EchoGéo est mis à disposition selon les termes de la licence Creative Commons Attribution - Pas d'Utilisation Commerciale - Pas de Modification 4.0 International 


\title{
La crise sociale aux Antilles françaises
}

Retour de la question sociale et reflux du politique

\author{
Justin Daniel
}

1 Inédits dans leur forme, les mouvements sociaux qui se sont déroulés aux Antilles françaises au début de l'année 2009, en menaçant de s'étendre à l'ensemble des départements d'outre mer, ont incontestablement été d'une ampleur, d'une durée et d'une intensité rarement égalées dans ces territoires à l'histoire sociale pourtant passablement mouvementée. Sans doute, des signes précurseurs d'une telle contestation étaient-ils perceptibles. Il en est ainsi de la mobilisation contre le coût excessif du carburant en Guyane à la fin de l'année 2008, avec un effet légèrement décalé en Guadeloupe et Martinique, de la dénonciation, sous une forme larvée mais persistante, de la hausse continuelle des prix, ou encore des inquiétudes suscitées par la crise mondiale et ses répercussions sur des économies fragiles, sans oublier une situation sociale de plus en plus dégradée. Mais cette contestation sociale a surtout agi comme le révélateur d'un malaise profond aux dimensions et racines multiples, dont la compréhension et l'examen requièrent, au-delà de la perception immédiate que l'on pourrait en avoir à travers une couverture médiatique elle même sans précédent, une analyse historicisée et contextualisée des situations antillaises. S'il est probablement prématuré de se livrer à un tel exercice dans le cadre de cette rubrique, il est néanmoins permis de dégager quelques enseignements qui sont autant de jalons pour de futures analyses. A cet égard, trois constats majeurs peuvent d'ores et déjà être établis: le premier procède du retour en force de la "question sociale » au sein des espaces publics antillais ; le seconde témoigne d'une forme de reflux du politique dans la gestion de la crise et fait advenir au grand jour une évolution des rapports entre l'outre mer et la métropole; enfin le troisième invite à s'interroger sur les modalités de sortie - en forme de trompe l'œil ? - de la crise.

\section{Le retour de la question sociale}

2 Sans conteste, ces mouvements sociaux consacrent le retour, d'une certaine manière métamorphosée ${ }^{1}$, de la question sociale, quelque peu occultée au cours de ces dernières 
années par un surinvestissement dans le champ des revendications identitaires. Rapportés à l'histoire récente, ils dévoilent du même coup l'ampleur du malaise qui sévit aux Antilles et l'inadéquation des solutions régulièrement apportées. En même temps, ils apparaissent comme des arènes où se sont concrétisées des stratégies élaborées par les différents acteurs dont l'analyse s'avère particulièrement riche d'enseignements sur la reconfiguration des espaces politiques et syndicaux.

Pour bien appréhender cette crise et mesurer sa portée, il convient de rappeler que la revendication d'égalité, qui était au fondement de la départementalisation intervenue à partir de 1946 a été, tout au long d'une histoire tissée de violences, le vecteur principal des luttes sociales aux Antilles. Couplée à une forte demande de reconnaissance et de dignité, elle s'est néanmoins systématiquement heurtée aux réticences du gouvernement central à mettre en œuvre le principe de l'identité législative ou l'extension des lois et règlements : une interprétation restrictive des textes - en particulier l'article 73 de la constitution de 1958 - a ainsi contribué à faire du cadre départemental un espace de luttes incessantes visant à imposer aux gouvernement successifs, peu enclins à céder, la prise en compte de la composante sociale de la citoyenneté. Faut-il rappeler, en effet, que l'égalité sociale entre les départements d'outre-mer et la métropole a été officiellement proclamée en 1996, soit cinquante ans après la loi qui la consacrait formellement? Elle s'est en outre accommodée de la pérennisation de rapports sociaux structurés par de profondes inégalités largement héritées d'un passé douloureux qui affleure en permanence les consciences. Au désenchantement généré par le processus contrarié de la départementalisation, s'ajoute donc le constat sans appel d'une situation sociale caractérisée par diverses formes de pauvreté, de précarité et d'exclusion (Daniel et alii, 2007) que ne parviennent pas à résorber les politiques publiques de développement. Cette situation présente au moins deux visages :

- celui, tout d'abord, de phénomènes plus diffus, plus étendus et plus intenses que dans l'Hexagone ainsi que de poches irréductibles d'une " pauvreté intégrée » (Paugam, 2005) mais persistante, en dépit de tous les dispositifs qui se sont succédé dans les DOM depuis plusieurs décennies. Ainsi subsistent aux Antilles des « îlots de population » qui conjuguent solidarité familiale et dépendance à l'égard des prestations sociales. Ces individus occupent une zone de pauvreté aux frontières labiles et fortement dépendantes des effets de seuils, côtoient les frontières de l'exclusion, y basculent parfois ; ils vivent le plus souvent aux marges du travail et des formes d'échange socialement consacrées : chômeurs de longue durée ayant parfois renoncé à chercher un emploi, allocataires du RMI, jeunes en quête d'emploi se promenant de stage en stage, de petits boulots en occupations provisoires. Significativement, le pourcentage de pauvres, en dépit des difficultés à saisir les contours d'une telle catégorie, atteignait 12,5\% en Guadeloupe et 12,1 \% à la Martinique en 2005, contre 6,1 \% dans l'Hexagone (Daniel et alii, 2005). De même, les DOM en général, et les Antilles en particulier, se caractérisent par l'importance du taux de bas revenu, même calculé en fonction d'un seuil spécifique tenant compte de l'écart avec le PIB de la métropole, et par un nombre très élevé de bénéficiaires de minimas sociaux, en particulier le RMI, soit, selon les données fournies par l'INSEE, 31592 bénéficiaires à la Martinique et 32052 en Guadeloupe au 31 décembre 2007 ;

- celui, ensuite, d'une nouvelle figure de pauvreté - correspondant à l'une des métamorphoses de la questions sociale - et perçue comme « désintégrante » à la fois par les professionnels de l'action sociale et la population. Elle met en scène des groupes d'individus, souvent abusivement assimilés à la catégorie générique des « jeunes », en voie de désaffiliation car 
privés de perspective et se reconvertissant dans des activités illicites les plaçant

délibérément en marge de la société et de tout dispositif efficient de prise en charge.

4 impasse sur le plan politique et statutaire : le constat quasi unanime d'un essoufflement du système de la départementalisation n'a d'égal que l'impossibilité à trouver une solution alternative, comme en témoignent les consultations populaires du 7 décembre 2003 (Daniel, 2006). Dans un tel contexte, trois éléments, au moins, se sont conjugués pour ériger la « question sociale » en enjeu majeur.

5 A commencer par le caractère transversal de la revendication principale articulée autour de la cherté de la vie et du pouvoir d'achat ; revendication qui a bénéficié d'emblée d'une forte légitimité au sein des populations antillaises. En effet, c'est peu dire que ce problème touche toutes les catégories sociales, mais qu'il affecte en particulier les plus démunies qui, comparativement à l'Hexagone, sont proportionnellement plus nombreuses. Dès lors, des catégories sociales traditionnellement peu portées à manifester leur mécontentement dans la rue, voire à prendre la parole, ont fini par franchir le pas en rejoignant dans la contestation des organisations plus traditionnelles ou en leur apportant un soutien explicite. réunissant des syndicats qui se sont forgés une identité forte à travers les lutes, des associations et différentes formes d'organisations présentant un front uni consacrant la centralité de la question sociale a été un puissant élément unificateur. Elle a favorisé la prise en charge, y compris après le déclenchement du mouvement, des revendications ou des attentes émanant des sociétés antillaises et permis d'agréger des mécontentements divers tout en évitant le piège des corporatismes ou des revendications purement catégorielles.

7 Toutefois, la forte mobilisation qui en a découlé n'est pas le fruit d'une simple conjoncture particulière. Elle se donne à voir comme la résultante de stratégies, parfois élaborées de longue date, selon des modalités distinctes en Guadeloupe et Martinique.

Dans le premier cas, le déclin politiquement attesté de la mouvance nationaliste sur le plan électoral à partir des années 90 a conduit cette dernière à déserter partiellement la scène politique pour investir le champ syndical. Transmuée en force de frappe syndicale, elle a constitué, à travers l'UGTG', l'épine dorsale du collectif $\mathrm{LKP}^{3}$ tout en réalisant une jonction réussie avec des mouvements culturels dont, en particulier le célèbre groupe AKIYO. Cette alliance est parfaitement incarnée par le LKP dans sa diversité; elle a également conféré une dimension identitaire à la contestation, sans que celle-ci ne supplante la question sociale ou ne remette en cause sa centralité, et encore moins ne trouve ou ne se donne pour objectif un prolongement directement politique.

Dans le second cas, la mouvance syndicale d'obédience trotskyste qui peine à être présente sur la scène politique et électorale martiniquaise, tout en ayant préféré depuis longtemps pénétrer des syndicats tels que la $\mathrm{CDMT}^{4}$ pour faire aboutir ses revendications immédiates, a joué un rôle majeur dans l'animation du mouvement et du «Collectif du 5 février ». Elle a ainsi contribué à occulter, au moins dans les médias, la présence des syndicats nationalistes - l'UGTM ${ }^{5}$ et la CSTM $^{6}$ - dont le rapport de proximité avec l'actuel pouvoir régional, dominé par les deux partis nationalistes que sont le $\mathrm{MIM}^{7}$ et le $\mathrm{CNCP}^{8}$, est apparu durant la phase active du mouvement comme un handicap9. Les revendications mises en avant cadrent mal avec l'objectif d'accession à la souveraineté, 
même si paradoxalement le mouvement nationaliste s'est depuis longtemps cantonné dans une gestion prudente des institutions décentralisées.

Dans les deux cas, le désinvestissement partiel ou provisoire du champ politicoidentitaire - espace privilégié de revendications au cours des dernières années - dans le cadre de cette contestation, tout en plaçant l'UGTM et la CSTM dans une position pour le moins inconfortable en Martinique, a puissamment favorisé le retour de la question sociale sous des formes et à partir de revendications qui sont entrées en résonance avec les attentes de la population. D'autant que le sentiment, déjà signalé, d'une certaine impuissance des acteurs politiques n'a pas peu incité les uns et les autres à s'en remettre, au moins dans un premier temps, aux "collectifs " pour se faire entendre, quittes à se retourner plus tard vers les instances politiques.

\section{Le reflux du politique}

11 L'une des caractéristiques majeures de ces mouvements sociaux, c'est sans aucun doute la dépossession des hommes politiques locaux de la parole, et l'apparition simultanée d'une parole débridée et libérée témoignant du foisonnement d'une société civile jusque là plus ou moins tenue en lisière D'un autre côté, la gestion de la crise par un Etat excentré et éloigné témoigne d'un changement significatif dans sa façon d'appréhender - ou de ne pas appréhender - les problèmes de l'outre mer.

Sur le premier point, il convient de rappeler que les relations entre les mouvements sociaux et les organisations politiques sont souvent complexes. Les premiers ont incontestablement une dimension politique et idéologique, agissent dans le domaine politique, mais ne sont pas politiquement structurés et ne posent que rarement la question du pouvoir (Denis, 2005). La remise en cause implicite du politique dans son fonctionnement routinier dont ils sont les porteurs est en même temps un témoignage inversé mais éloquent de l'intérêt que beaucoup de citoyens continuent à accorder à la chose publique. De même, nul ne saurait nier que la contestation en Guadeloupe et Martinique des rapports de domination, à partir parfois d'une réactivation des tensions socio-raciales ou d'une polarisation ethnique et de la mise en scène d'identités forgées par les différents groupes constitutifs des sociétés antillaises, est éminemment politique. Mais une telle contestation n'a jamais porté en elle même son propre dépassement sur le plan politique, en dépit de sa radicalité dans la formulation des revendications et de son appel à une véritable refondation, au-delà des relations de travail - point de cristallisation des tensions socio-raciales - des sociétés antillaises: elle s'est inscrite d'emblée dans une logique classique de recours à l'Etat, tant comme instance d'allocations de ressources que comme instance de régulation de rapports sociaux (Larcher, 2009) ${ }^{10}$. Du même coup, elle a contribué à disqualifier, non pas tant le personnel politique local ravalé au rang de médiateurs ou de facilitateurs dans les négociations ${ }^{11}$, que son discours et sa parole rendus inaudibles, parce que totalement déphasés par rapport aux attentes et aux souhaits du moment: la convocation des outils de développement tels que le Schéma régional de développement économique pour la Guadeloupe, le Schéma martiniquais de développement économique et l'Agenda 21 pour la Martinique, brandis ça et là comme des réalisations majeures, ainsi que la focalisation sur une éventuelle évolution institutionnelle, voire la création d'une nouvelle collectivité territoriale sur la base de l'article 74 de la Constitution, ne pouvaient être d'aucun secours. Tous ces éléments ne 
sont pas de nature à établir un quelconque lien avec les revendications défendues par les « collectifs » et massivement soutenues par les populations.

13 En outre, la multiplication d'arènes de débats et de mise en visibilité du mouvement ont définitivement supplanté les espaces traditionnels d'expression politique, privant les élus de leur traditionnelle capacité d'intervention tout en libérant la parole populaire. Celle-ci a parfois dessiné en creux des modèles alternatifs fondés sur une vision quelque peu irénique d'un futur incertain, en s'appuyant sur la réactivation d'anciens réseaux de solidarité, d'entraide familiale, de voisinage et associative, accréditant l'idée que le système actuel - et ce terme englobe de manière plus générale le modèle de développement à l'œuvre depuis plusieurs décennies dans ces territoires - n'est plus viable ou a atteint ses limites et qu'il convient de le repenser. Ce qui revient à souligner l'impuissance des élus à concevoir et à mener à bien un tel projet.

D'un autre côté, le rôle tenu par l'Etat et les attentes qu'il nourrit appellent plusieurs remarques. En premier lieu et de manière générale, son rapport avec l'outre-mer est incontestablement en train de changer. Une nouvelle génération de responsables politiques, dont la sociographie révèle qu'ils connaissent mal les problèmes et les préoccupations qui se font jour dans tout l'outre-mer, a émergé en métropole, à droite comme à gauche. Son intérêt pour ces régions éloignées - lieu de condensation de problèmes divers et complexes - au sein desquelles elle se trouve parfois privée de réseaux solidement constitués, est manifestement moins marqué, comparativement à celle qui l'a précédée. Il n'est pas certain, en sens inverse, que cette évolution soit clairement perçue dans les différents territoires concernés. Là résident sans doute de nombreux malentendus ou incompréhensions, source parfois de maladresses. On ne peut qu'être frappé de l'attitude du Secrétaire d'Etat à l'outre-mer qui a donné l'impression de découvrir l'ampleur de certains problèmes et leur épaisseur historique, avant d'endosser l'habit du nouveau converti. On ne peut qu'être surpris par le rôle qui continue à être assigné à l'Etat, en dehors des dossiers relevant directement de sa compétence, dans le règlement des conflits à un moment où l'appel en faveur de l'exercice de nouvelles responsabilités semble émaner de toute part. D'où d'ailleurs les hésitations observées au niveau du gouvernement, entre un secrétaire d'Etat tenté de s'engager sur des dossiers exigeant des arbitrages interministériels, un Président de la République resté longtemps silencieux, contrairement à ses habitudes, et un premier ministre soucieux de reprendre en main le dossier tout en se défaussant sur deux médiateurs.

C'est dire que la gestion de l'outre-mer depuis Paris s'avère de plus en plus compliquée et explique, au-delà des tergiversations, la difficulté à élaborer localement un dispositif de sortie de crise mobilisant des partenaires sociaux peu enclins à dialoguer, des élus locaux longtemps restés inaudibles et un pouvoir central perçu comme de plus en plus éloigné des réalités ultramarines.

\section{Une sortie de crise en trompe l'œil ?}

16 A y regarder de plus près, la sortie de crise a été organisée autour de trois dispositifs centraux autour desquels s'agrègent toute une série d'acquis à caractère plus ou moins secondaire. Le premier résulte de la satisfaction des deux revendications principales relatives à la baisse des prix d'une sélection de produits de première nécessité d'une part, et d'autre part, de l'augmentation de 200 euros des salaires les plus bas. Au-delà des modalités complexes de sa mise en œuvre, cette dernière mesure fait intervenir, non 
seulement les employeurs, mais aussi l'Etat qui a mis en place par anticipation, contrairement au choix initialement arrêté lors de l'adoption de la loi, le RSA (revenu de solidarité active) provisoirement transmué en RSTA (revenu supplémentaire temporaire d'activité). De même, les régions et les départements ont consenti, sans doute par souci d'éviter l'enlisement du conflit et d'accélérer son règlement, à financer partiellement sur une période d'un an, les salaires dans le secteur privé. Un tel dispositif pose inévitablement le problème de sa pérennisation et de la capacité des petites entreprises, notamment celles qui sont très petites et proportionnellement les plus nombreuses, à le maintenir au-delà de la période probatoire. A cela s'ajoute le risque pris par les collectivités concernées d'ouvrir une véritable boîte de Pandore pour l'avenir. Surtout, le dispositif en question ne peut constituer en lui même un substitut à des solutions structurantes s'inscrivant dans la durée. Car l'un des mérites de ces mouvements sociaux a été de révéler un malaise profond débordant le cadre des revendications et des préoccupations à court terme telles qu'elles se sont exprimées: derrière les attentes immédiates se cachent en réalité des problèmes structurels dont les solutions appellent sans doute des révisions importantes des politiques publiques.

Or le deuxième dispositif de sortie de crise, le projet de loi pour le développement économique de l'outre-mer (LODEOM), dont l'adoption a été longtemps retardée avant de connaître une brutale accélération, ne semble pas avoir été conçu dans une telle perspective. Quoiqu'il continue à afficher, après avoir subi quelques correctifs pour tirer les conséquences de la crise qui a sévi aux Antilles, une volonté de " rupture », il s'inscrit en réalité dans la plus stricte continuité des politiques publiques de développement expérimentées depuis plusieurs décennies dans les DOM. L'une de ses dispositions centrales, la création de zones franches globales d'activités, pourrait en effet jouer le rôle de « locomotive de la croissance » qui lui est assignée, sans pour autant remettre en cause ni les déséquilibres structurels qui minent les économies antillaises ni les inégalités profondes qui structurent les rapports sociaux et engendrent de graves tensions.

Enfin, le troisième dispositif est bien sûr constitué par les « Etats généraux » proposés par le président Sarkozy lors de son intervention télévisée du 19 février 2009 et dont le but immédiat était d'aider à désamorcer la crise tout en permettant de gagner du temps. Outre le fait que l'expression utilisée en la circonstance pose problème dans la mesure où il s'agit d'« Etats généraux » qui procèdent non pas d'initiatives populaires, mais du haut, après avoir été d'emblée cadrés à partir d'un certain nombre d'axes thématiques préalablement définis, une telle solution ne peut être efficace qu'à une double condition : d'une part, elle doit permettre la libre expression de légitimités concurrentes afin d'éviter le piège de la simple réhabilitation d'une sphère officielle dont on a vu qu'elle a subi un processus de disqualification constituant un signal fort auquel nul ne saurait rester sourd; d'autre part, elle doit aborder sans tabou aucun des débats propres à nourrir des propositions de réformes structurelles, susceptible de remettre en cause bien des situations, afin de conjurer le risque d'apparaître comme une grand-messe sans lendemain. Autant dire que ce pari est loin d'être gagné. 


\section{BIBLIOGRAPHIE}

Castel, Robert. Les métamorphoses de la question sociale : une chronique du salariat, Paris:

Fayard, (L'espace du politique), 1995.

Daniel, Justin, Patrick Bruneteaux, Joelle Kabile, et al. « Précarité, pauvreté et formes spécifiques d'exclusion à la Martinique : une approche qualitative,Rapport remis au Ministère de l'outre-mer en décembre 2006 ». Paris: Ministère de l'Outre-mer, 2007.

Daniel, Justin. « Les élus face à la réforme institutionnelle et à l'acte II de la décentralisation : la difficile conciliation d'aspirations contradictoires » in Thierry Michalon (ed.), Entre assimilation et émancipation : l'outre-mer français dans l'impasse, Rennes: Editions les Perséides, 2006.

Denis, Serge. L'action politique des mouvements sociaux d'aujourd'hui. Le déclin du politique comme procès de politisation? Québec, Les Presses de l'Université Laval, 2005

Larcher, Silyane. « Les Antilles françaises ou les vestiges de l'Empire ? Les aléas d'une citoyenneté sociale outre-mer ", laviedesidees.fr, le 20 février 2009.

Paugam, Serge. Les formes élémentaires de la pauvreté, Paris: Presses universitaires de France, (Le lien social), 2005.

\section{NOTES}

1. Pour reprendre une formule dérivée du titre de l'ouvrage de Robert Castel (1995).

2. / Union Générale des Travailleurs de Guadeloupe

3. Liyannaj Kont' Pwofitasyon (LKP : rassemblement contre les abus)

4. Centrale Démocratique Martiniquaise des Travailleurs.

5. Union Générale des Travailleurs de Martinique

6. Centrale Syndicale des Travailleurs Martiniquais

7. Mouvement Indépendantiste Martiniquais.

8. Conseil National des Comités Populaires.

9. Le président de la région Martinique, dont le malaise a été perceptible dès le début du mouvement a quitté les négociations et a refusé de participer à la signature du protocole de sortie de crise, estimant que la méthode utilisée n'était pas appropriée.

10. . On notera que pour l'essentiel, les négociations se sont déroulées, de manière symbolique, dans les deux préfectures, ce qui correspond à une vieille tradition locale qui témoigne de la forte polarisation des rapports sociaux et du caractère déficient du dialogue social aux Antilles.

11. Rôle que les élus ont accepté de jouer, aidant à bien des égards, à débloquer la situation. 


\section{RÉSUMÉS}

La crise sociale qui a récemment secoué les Antilles françaises consacre le retour de la « question sociale » sur les scènes politiques insulaires. Elle s'est également traduite par un dessaisissement du personnel politique dont la parole est devenue inaudible, et une montée en puissance de la société civile. Loin d'avoir réglé les problèmes structurels, elle témoigne cependant d'une évolution significative des rapports entre l'outre-mer et l'Hexagone.

The social crisis which recently shook the French West Indies legitimises the return of the "social question" on the insular political scenes. It also resulted in a withdrawal of the political personnel whose discourse became inaudible and in the emergence of civil society. Far from having resolved the structural problems, it reveals a significant change of the relationship between the overseas territories and the Hexagon.

\section{INDEX}

Mots-clés : mouvement social, organisations politiques, Etat, nationalisme.

Keywords : social movement, political organizations, State, nationalism.

\section{AUTEUR}

\section{JUSTIN DANIEL}

Justin Daniel est professeur de science politique et Doyen de la Faculté de droit et d'économie de la Martinique. Il est également membre Centre de recherche sur les pouvoirs locaux dans la Caraïbe (CRPLC, UMR CNRS 8053). Il a récemment publié :

- 2008 : « La citoyenneté inachevée : une analyse comparative des situations antillaises et portoricaine », in Nancy L. Green et Marie Poinsot Histoire de l'immigration et question coloniale en France, Paris, la Documentation française-Centre national de l'histoire de l'immigration, 2008, p. 139-144.

- 2008 : « Obligations de service public et continuité territoriale entre les DOM-ROM et la métropole : des dispositifs en trompe-l'œil ?», in Roselyne Allemand et Laurence Solis-Potvin, Egalité et non discrimination dans l'accès aux services publics et politiques publiques territoriales, Paris, L'harmattan (GRALE).

- 2007 : L'outre-mer a l'épreuve de la décentralisation : Nouveaux cadres institutionnels et difficultés d'adaptation (ed.), Paris, l'Harmattan. 